\title{
Changes in Tear Proteome After Acupuncture Treatment in Dry Eye
}

\author{
Louis Tong $\mathbb{D}^{1-4}$ \\ Lei Zhou ${ }^{3-5}$ \\ Siew Kwan Koh ${ }^{5}$ \\ Yan $\mathrm{Gao}^{5}$ \\ Hla Myint Htoon ${ }^{1,3}$ \\ AiHua Hou ${ }^{1,3}$ \\ Lu Deng ${ }^{6}$ \\ Qi-Ping $\mathrm{Wei}^{7}$ \\ Pat Lim ${ }^{8}$
}

'Ocular Surface Research Group, Singapore Eye Research Institute,

Singapore; ${ }^{2}$ Corneal and External Eye

Disease Service, Singapore National Eye

Centre, Singapore; ${ }^{3}$ Eye-Academic

Clinical Program, Duke-National

University of Singapore (NUS) Medical

School, Singapore; ${ }^{4}$ Department of

Ophthalmology, Yong Loo Lin School of

Medicine, NUS, Singapore; ${ }^{5}$ Ocular

Proteomics Platform, Singapore Eye

Research Institute, Singapore;

${ }^{6}$ Department of Statistics and Applied

Probability, Faculty of Science, NUS,

Singapore; ${ }^{7}$ Beijing University of Chinese

Medicine, Beijing, People's Republic of

China; ${ }^{8}$ Singapore Chung Hwa Medical

Institution, Singapore

\section{Introduction}

Dry eye, a prevalent, multi-factorial disease of the tear and ocular surface affects $14 \%$ to $33 \%$ of adults worldwide and is more common in Asia. Because mild to moderate forms of dry eye are so common, they incur tremendous economic and health burden. Dry eye can result from increased tear instability or less commonly, reduced tear production. Despite the fact that dry eye etiology is heterogenous, common disease hallmarks are raised tear osmolarity and inflammation. Therapies for dry eye may not obtain satisfactory results. A common approach is to control aggravating factors and instill artificial tear in mild-moderate dry eye disease, which accounts for majority of dry eye cases. ${ }^{1}$

Traditional Chinese Medicine (TCM) is a system of healthcare that considers the human body as a unified system, and disharmony results in disease. Acupuncture is one of the modalities in TCM, but knowledge on the biological mechanism of this therapy in dry eye is lacking. A previous report in this journal investigated tear proteomics changes after acupuncture, and evaluation of the changes by functional annotation of the proteins help to elucidate the mechanism of action of acupuncture. ${ }^{2}$ However, this type of longitudinal analysis of global tear proteins has not been published again, and the only other such longitudinal proteomics analysis evaluated dry eye treatments with diquafosol or cyclosporine. ${ }^{3}$ Longitudinal proteomics analysis is hypothesis-free and may offer a glimpse into the mechanism of action of the treatment in complex diseases.

The aim of this report is to further investigate changes in tear proteomics profile after acupuncture in dry eye patients longitudinally and to understand how this treatment may influence the pathology of dry eye.

\section{Methods}

\section{Study Design}

This was a prospective case series with samples from a subgroup of patients in a randomized interventional trial (clinicaltrials.gov, NCT02219204, registered 14 Aug 2014, started recruitment on Mar 2015 and ended recruitment on Dec 2015.)

Approval was obtained from the central Institutional Review Board (IRB) of Singapore Health services, and the study adhered to the tenets of the Declaration of Helsinki for human research. Written informed consent was obtained from participants, and they had demonstrated lung-kidney yin deficiency by a questionnaire, and the inclusion, exclusion criteria, method of randomization of participants, group 
assignment, study procedures and clinical efficacy results have been previously published. ${ }^{4}$ For ease of reference, these details are summarized in eSupplementary Table 1. The dry eye criteria was dry eye symptom (Standardised Patient Evaluation of Eye Dryness) score $>6$, and signs of dry eye (tear break-up time $<10 \mathrm{~s}$ or Schirmer's test $<10$ $\mathrm{mm} / 5 \mathrm{~min}$ ) and any corneal fluorescein dye staining. We did not include cases of severe meibomian gland dysfunction where there was one or more visible meibomian plugs, or if the meibum secretions were inspissated. However, mild cases (where majority of glands were clinically expressible) were included. From the original study, 15 participants were randomly selected from participants using Systane Ultra Eyedrops, and another 15 from those with acupuncture performed and also use Systane Ultra Eyedrops.

\section{Tear Protein Extraction and LC-MS Analysis}

The Schirmer strips were halved along its length and one half used for tear proteomics analysis. Elution of proteins was performed as described previously. ${ }^{5}$ Briefly, the halved Schirmer's strip was cut into tiny fragments and incubated in $50 \mathrm{mM}$ ammonium bicarbonate solution containing protease inhibitor. ITRAQ 6-plex was then performed. Around $50 \mu \mathrm{g}$ of tear protein from each sample was used for digestion and labelling based on iTRAQ protocol. Briefly, tear protein sample was denatured using $10 \%$ sodium lauryl sulfate (SDS) first and reduced using $50 \mathrm{mM}$ tris (2-carboxyethyl) phosphine (TCEP) $\left[60^{\circ} \mathrm{C}, 1\right.$ hour]. Reduced protein samples were pipetted into a membrane cartridge (Pall Corporation, Port Washington, NY, US) which was cut off at $30 \mathrm{kDa}$. The cartridge was then centrifuged twice with $200 \mathrm{ul} 75 \%$ urea in $0.1 \mathrm{M}$ tris $\mathrm{HCl}, \mathrm{pH} 7.6$ at $14,000 \mathrm{~g}$ for $15 \mathrm{~min}$. Cysteine blocking was performed using $0.5 \mathrm{M}$ iodoacetamide (IAA) in $75 \%$ urea solution. The mixture was incubated in the dark $(20$ $\mathrm{min}$ ), followed by centrifugation three times with $100 \mu \mathrm{L}$ $75 \%$ urea solution and $50 \mathrm{mM}$ ammonium bicarbonate, respectively. Trypsin (SCIEX, Framingham, MA, US) was added at $16 \mathrm{~h}$ at $37^{\circ} \mathrm{C}$ to digest the samples on-cartridge. This was followed by elution of peptides with $40 \mu \mathrm{L}$ of $50 \mathrm{mM}$ ammonium bicarbonate and $50 \mu \mathrm{L} 0.5 \mathrm{M}$ sodium chloride. The elution was dried using vacuum concentrator.

Dried peptides were added to $20 \mu \mathrm{L}$ of 0.5 $\mathrm{M}$ triethylammonium bicarbonate (TEAB) and iTRAQ reagents $113,114,115,116,117$ and 118. The mixtures were incubated at room temperature ( 2 hours). Labeled samples were combined and cleaned up using strong cation exchange cartridge (SCIEX) and ultramicro spin columns (The Nest Group, Southborough, MA, US) before nano LC-MS/MS analysis.

Peptide samples were constituted in $0.1 \%$ formic acid in water for injection and analysed using Dionex Ultimate 3000 nano-LC (Thermo Fisher Scientific, Waltham, MA, US) coupled with Triple TOF 5600 mass spectrometer (SCIEX). Sample was loaded onto a $75 \mu \mathrm{m} \times 2 \mathrm{~cm}$ Acclaim PepMap 100 C18 trap column (Thermo Fisher Scientific), using flow rate of $5 \mu \mathrm{L} / \mathrm{min}$. After washing 5 min with $0.1 \%$ formic acid in $2 / 98(\mathrm{v} / \mathrm{v})$ of acetonitrile/ water, the system was switched into a $75 \mu \mathrm{m} \times 15 \mathrm{~cm}$ Acclaim PepMap RSLC C18 column (Thermo Fisher Scientific). Mobile phase A was $0.1 \%$ formic acid in $2 \%$ acetonitrile $(\mathrm{v} / \mathrm{v})$ and mobile phase B was $0.1 \%$ formic acid in $98 \%$ acetonitrile $(\mathrm{v} / \mathrm{v})$. The gradient profile was $5 \%$ $\mathrm{B}$ at $0 \mathrm{~min}, 12 \% \mathrm{~B}$ at $45 \mathrm{~min}, 40 \% \mathrm{~B}$ at $100 \mathrm{~min}$ and $90 \%$ $\mathrm{B}$ at $120 \mathrm{~min}$ to $130 \mathrm{~min}$, and the flow rate used was 0.3 $\mu \mathrm{L} / \mathrm{min}$. The analysis column, linked to a spray tip (New Objective, Woburn, MA, US), was in turn coupled to the nano-spray interface of tripleTOF $5600 \mathrm{MS}$.

Information dependent acquisition (IDA) mode was used for data collection by Analyst TF 1.7 (SCIEX). The accumulation time of TOFMS scan was $250 \mathrm{~ms}(\mathrm{~m} / \mathrm{z}$ : 400-1250), followed by 40 product ion scans, each 50 $\mathrm{ms}(\mathrm{m} / \mathrm{z}: 100-1500)$. This procedure included only ions with charge of 2-5 and abundance above 120 counts. We excluded former target ions $12 \mathrm{~s}$ after one occurrence.

ProteinPilot 5.0 (SCIEX) was used to process the raw data. Protein level was expressed as a ratio of protein intensity at week 4/week 0 for each participant.

\section{Statistical Analysis}

Hierarchical clustering (supervised) and illustration with heat mapping were used to illustrate global protein change. A forest plot was constructed to illustrate the changes of 10 previously reported tear proteins. Statistical significance of changes in tear proteins was defined as $p$ value of less than 0.001 (to account for effect of multiple testing); however, any $\mathrm{p}<0.01$ would also be noted.

\section{Results}

This report involved participants who had eight sessions of acupuncture within four weeks. Tear samples were obtained at baseline and at the end of the fourth week. The heat map (Figure 1) representing differentially expressed proteins demonstrated that around 2/3 of the overall tear proteins 


\section{Responsive Unresponsive LUB ACP LUB ACP}
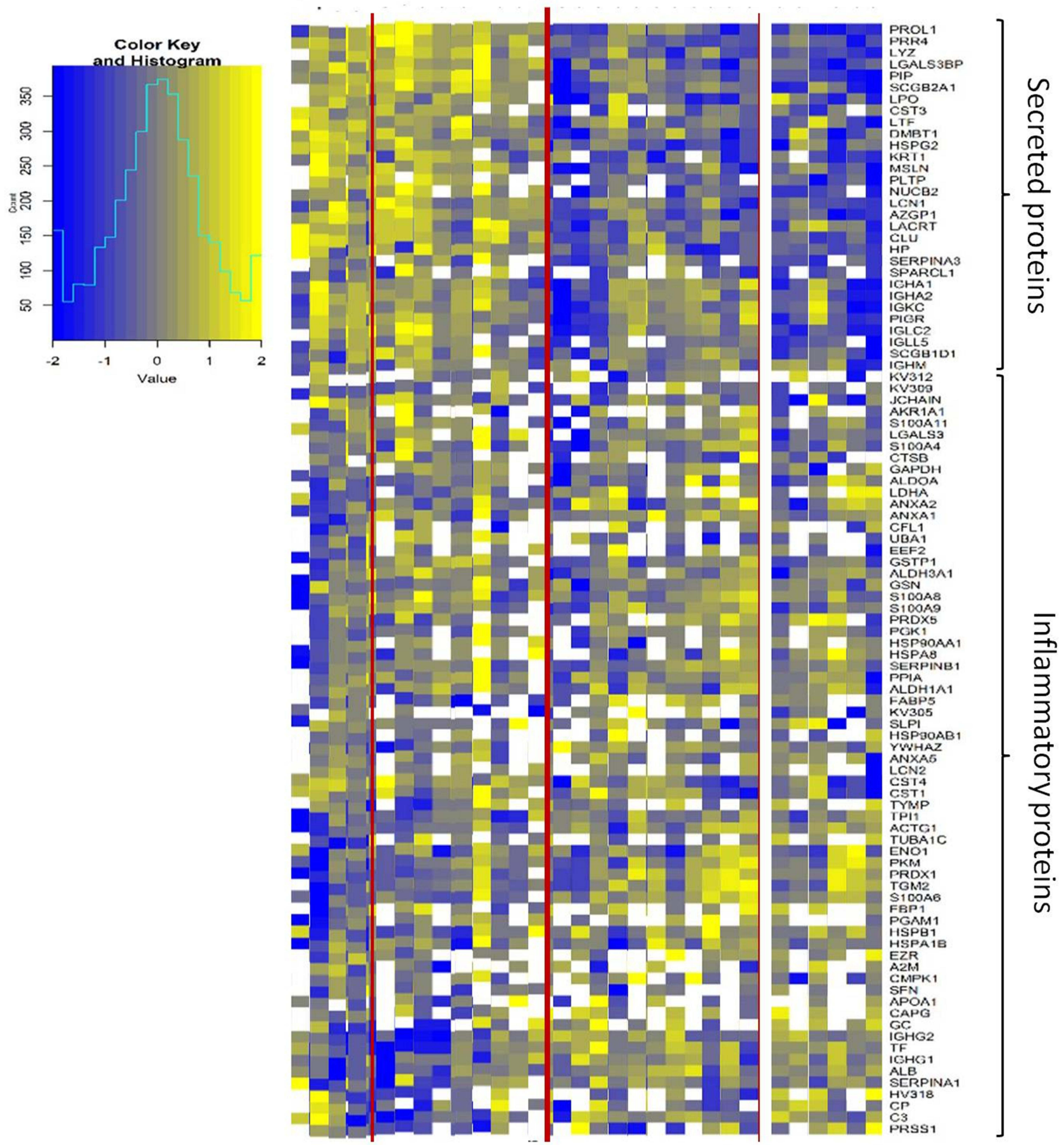

Figure I Heat maps showing the result of hierarchical clustering of the changes in tear proteins. Each row represents a tear protein. Secreted proteins are shown in the top, and inflammatory proteins are shown in the bottom of the heat map. A blue color represents a decrease in the tear protein, whereas a yellow color represents an increase in the tear protein after treatment, compared to baseline readings. Participants (columns), ACP: acupuncture with artificial tear, LUB: only artificial tear. White squares: tear protein not detected. Only proteins with missing values less than $50 \%$ of the time were displayed. 
were within a cluster that is known to be proinflammatory in nature from the literature, whereas $1 / 3$ of the proteins were known to be supportive of the ocular surface, of which many representing tear proteins secreted from the lacrimal gland or ocular surface epithelial cells. The supportive protein group includes members like lacritin and lipocalin, whereas the inflammatory group includes S100A6, 8, 9 as well as transglutaminase-2. Many of the inflammatory proteins were upregulated in dry eye patients whereas the supportive proteins decreased, compared to normal people. ${ }^{5}$ A "responsive" tear protein change was defined as an increase in supportive proteins and decrease in inflammatory proteins whereas a non-favorable change was conversely defined. Nine out of fifteen participants who underwent acupuncture showed a "responsive" pattern of protein change with an increase in the secretory proteins, whereas only four out of fifteen participants treated with Systane Ultra alone showed such a pattern of protein change. Both groups of participants were treated with four times a day Systane Ultra, which was a transient gel that potentially could entrap tear proteins.

Using a targeted approach, the alteration of 10 tear proteins implicated in dry eye from previous study ${ }^{6}$ was examined (Figure 2). There was a tendency to increase the tear levels of lysozyme, prolactin-induced protein and S100A11 in the patients treated with acupuncture $(n=$ $15)$, whereas the use of Systane Ultra alone $(n=15)$ tends to reduce the concentration of these proteins. However, these changes are not statistically significant. The tendency for these changes in global proteomic signatures suggests that these assays may be more informative than changes of individual proteins.

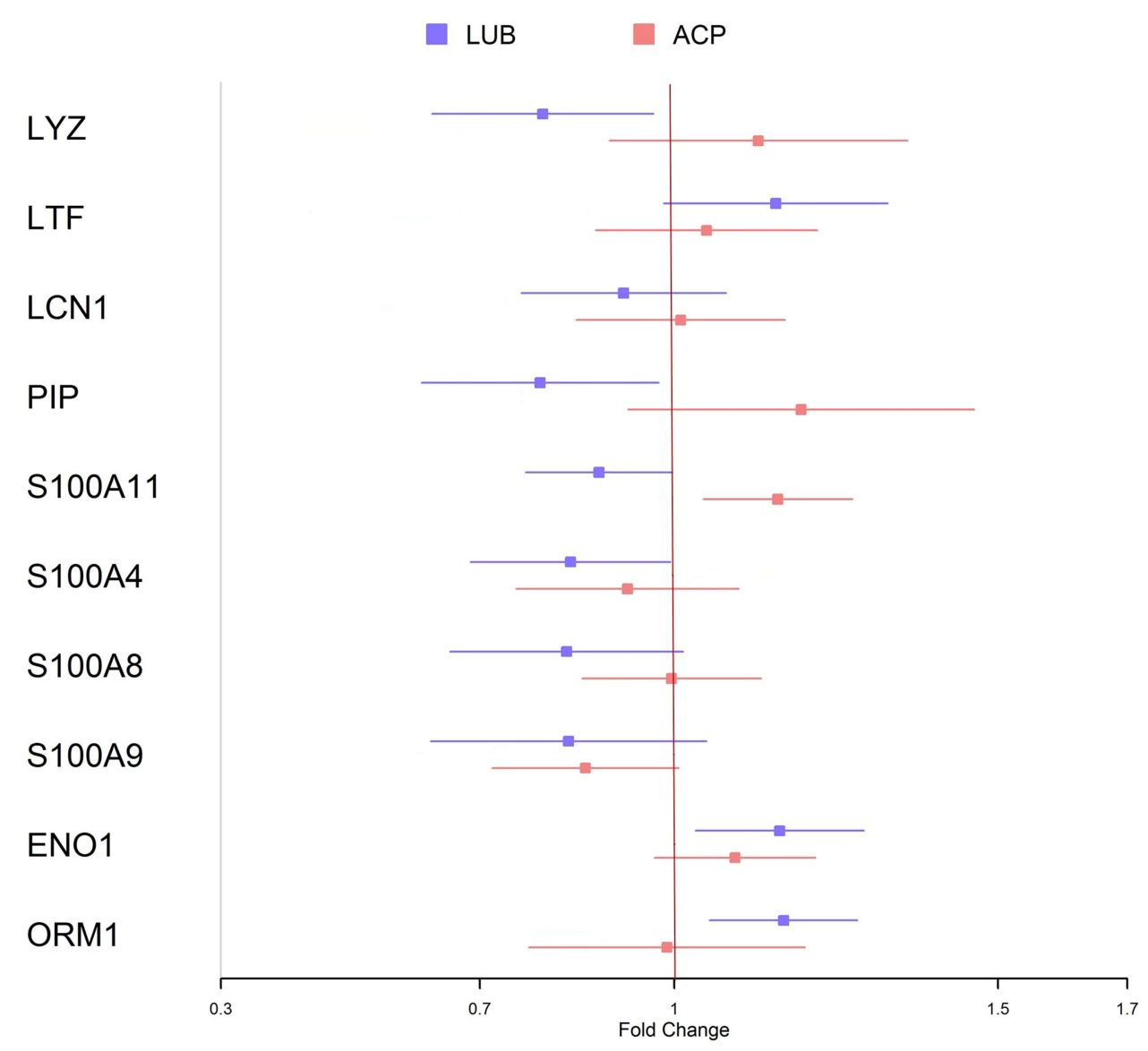

Figure 2 Forest plots illustrating the change in the levels of the 10 major tear proteins previously implicated in dry eye. The colored symbols indicate mean of the fold change, and the error bars indicate standard errors of means. The four protein at the top of the plots represent proteins decreased in dry eye whereas the others are known to be elevated in dry eye. Change of $>I$ indicates greater than baseline levels at the end of one month, whereas a change of less than I indicates a reduction from baseline level.

Abbreviations: LUB, Systane Ultra eyedrop only; ACP, Acupuncture with Systane Ultra; LYZ, lysozyme; LTF, lactoferrin; LCN, lipocalin; PIP, prolactin-induced protein; ENO, enolase; ORM, orosomucoid. 
The participants in the two treatment groups did not differ in any of their baseline clinical parameters (eSupplementary Table 2). The mean age of the participants in the acupuncture group $(\mathrm{n}=15)$ was $56.9 \pm 7.9$ years, whereas that in the Systane Ultra only group $(n=15)$ was $60.3 \pm 8.0$ years. One out of fifteen in the acupuncture group and five out of fifteen in the Systane Ultra only group were male. The mean SPEED (symptom score) were $13.7 \pm 4.1$ and $12.7 \pm 4.8$ in the acupuncture and Systane Ultra only group respectively. The tear break up times were $2.3 \pm 0.7 \mathrm{~s}$ and $1.8 \pm 0.6$ $\mathrm{s}$ respectively. The Schirmer test results were $11.8 \pm 6.6 \mathrm{~mm}$ and $15.3 \pm 7.2 \mathrm{~mm}$ respectively, suggesting these were not cases with aqueous deficient dry eye. Clinical characteristics of the participants at baseline were not predictive of the pattern of change in the tear proteins [data not shown].

\section{Discussion}

Around 400 tear proteins were relatively quantified using iTRAQ technique and after one month of treatment with acupuncture in dry eye, there was a tendency for tear secretory proteins to increase. Acupuncture may increase tear levels of three of these individual proteins but these changes were short of statistical significance.

Our findings are supported by the other dry eye study using longitudinal proteomics which showed that after two months of acupuncture, the tear protein counts of secretory proteins tripled, and cytosolic proteins decreased significantly. The authors reported that $30 \%$ of the 169 upregulated proteins were secretory proteins, whereas the most common downregulated proteins $(44 \%$ of the 142 proteins) were cytosolic proteins. Among the upregulated secreted proteins reported by the authors were immunoglobulin light chains, transferring and clusterin, which are also in the secretory protein group in our study (Figure 1). The authors suggested that after treatment, more fluid was secreted from the intracellular space into the tear. ${ }^{2}$

Another study compared the changes in tear proteomic signature before and after treatment with either diquafosol or cyclosporine. Interestingly, the heat map analysis showed that diquafosol-induced changes were more marked than cyclosporine-induced changes. Since diquafosol acts through increased aqueous tear and mucin secretion, the study suggests that tear proteomic profiling may detect changes in secretion more easily than reduction in inflammatory mediators. ${ }^{3}$

A previous study of acupuncture showed that it increased tear lactoferrin concentration, at least transiently. ${ }^{7}$ Lactoferrin, a multifunctional protein, has anti-microbial and anti-inflammatory effects, and is known to be decreased in the tear in dry eye. It was unclear if those patients ${ }^{7}$ also had yin-deficiency.

A rabbit study has found tear lipocalin to increase after acupuncture. ${ }^{8}$ A second rabbit study showed changes in the lacrimal gland morphology after acupuncture suggestive of increased tear secretion. ${ }^{9}$

The current study is small, designed as a prospective case series (sampled from a larger study) and is not powered to evaluate tear protein changes. The trend of changes in the lacrimal proteins suggests that acupuncture may induce an increase in tear production. It is not possible to determine from the data whether this increase was primarily a reflex or a basal production, or secondary to reduced inflammatory damage to the secreting glands. In any case, we show that global tear protein profile changed after therapy, suggesting that tear protein manipulation may have wider significance as a therapeutic strategy in dry eye.

Some modalities of dry eye treatment such as nasostimulation also act mechanistically through increased tear production. It would be interesting to see if these modalities induce a similar pattern of tear protein change as acupuncture.

\section{Conclusion}

In summary, we found that acupuncture treatments over one month in dry eye may induce a favorable increase in secretory proteins in the tear fluid, over and beyond a transient gel that retains tear. This has implications for the mechanism of acupuncture in dry eye.

\section{Acknowledgments}

The study is supported by grants from the Ministry of Health, Singapore MOH TCMCRG, and National Medical Research Council NMRCICSA $1017 \backslash 2017$. The authors would like to thank SingHealth Foundation for supporting proteomics core facility at Singapore Eye Research Institute and Singapore National Medical Research Council's (NMRC) Centre Grants to the Singapore Eye Research Institute.

\section{Disclosure}

The authors have no conflicts of interest in this work.

\section{References}

1. Craig JP, Nichols KK, Akpek EK, et al. TFOS DEWS II definition and classification report. Ocul Surf. 2017;15(3):276-283. doi:10.1016/j. jtos.2017.05.008 
2. Liu Q, Liu J, Ren C, et al. Proteomic analysis of tears following acupuncture treatment for menopausal dry eye disease by two-dimensional nano-liquid chromatography coupled with tandem mass spectrometry. Int $J$ Nanomedicine. 2017;12:1663-1671. doi:10.2147/IJN.S126968

3. Ji YW, Kim HM, Ryu SY, et al. Changes in human tear proteome following topical treatment of dry eye disease: cyclosporine a versus diquafosol tetrasodium. Invest Ophthalmol Vis Sci. 2019;60 (15):5035-5044. doi:10.1167/iovs.19-27872

4. Tong L, Htoon HM, Hou AH, et al. Acupuncture and herbal formulation compared with artificial tears alone: evaluation of dry eye symptoms and associated tests in randomized clinical trial. BMJ Open Ophthalmol. 2018;3(1):e000150. doi:10.1136/bmjophth-2018-000150

5. Tong L, Zhou L, Beuerman R, et al. Effects of punctal occlusion on global tear proteins in patients with dry eye. Ocul Surf. 2017;15 (4):736-741. doi:10.1016/j.jtos.2017.04.002
6. Zhou L, Beuerman RW, Chan CM, et al. Identification of tear fluid biomarkers in dry eye syndrome using iTRAQ quantitative proteomics. J Proteome Res. 2009;8(11):4889-4905. doi:10.1021/ pr900686s

7. Shi JL, Miao WH. [Effects of acupuncture on lactoferrin content in tears and tear secretion in patients suffering from dry eyes: a randomized controlled trial]. Zhong Xi Yi Jie He Xue Bao. 2012;10(9):1003-1008. doi:10.3736/jcim20120909. Chinese.

8. Qiu X, Gong L, Sun X, et al. Efficacy of acupuncture and identification of tear protein expression changes using iTRAQ quantitative proteomics in rabbits. Curr Eye Res. 2011;36(10):886-894. doi: $10.3109 / 02713683.2011 .601843$

9. Gong L, Sun X. Treatment of intractable dry eyes: tear secretion increase and morphological changes of the lacrimal gland of rabbit after acupuncture. Acupunct Electrother Res. 2007;32(3-4):223-233. doi:10.3727/036012907815844011
Clinical Ophthalmology

\section{Publish your work in this journal}

Clinical Ophthalmology is an international, peer-reviewed journal covering all subspecialties within ophthalmology. Key topics include: Optometry; Visual science; Pharmacology and drug therapy in eye diseases; Basic Sciences; Primary and Secondary eye care; Patient Safety and Quality of Care Improvements. This journal is indexed on PubMed

\section{Dovepress}

Central and CAS, and is the official journal of The Society of Clinical Ophthalmology (SCO). The manuscript management system is completely online and includes a very quick and fair peer-review system, which is all easy to use. Visit http://www.dovepress.com/ testimonials.php to read real quotes from published authors. 